\title{
EVALUATION OF AUTOMATED CELL COUNTS IN BODY FLUIDS USING SYSMEX XT-4000I - AN IMPORTANT NEED OF THE DAY!
}

\author{
Umadevi Komarla Parthasarathi', Sujay Prasad², Jayaram³, Govardhan Jagadeesh Kumar4 \\ ${ }^{1}$ Assistant Professor, Department of Pathology, Aarupadai Veedu Medical College, Puducherry. \\ 2Senior Consultant Pathologist, Department of Pathology, Anand Diagnostic Laboratory, Bangalore. \\ ${ }^{3}$ Senior Consultant Pathologist, Department of Pathology, Anand Diagnostic Laboratory, Bangalore. \\ ${ }_{4}^{4}$ Assistant Professor, Department of Dermatology, Aarupadai Veedu Medical College, Puducherry.
}

\section{ABSTRACT}

Cell counts in body fluids provide information to clinicians for diagnosis and treatment of a wide variety of medical conditions. Routinely, the analysis of cell counts is performed manually using a haemocytometer due to numerous limitations in manual inspection and counting. Automated counters are therefore employed to perform the test faster and with more accuracy.

\section{OBJECTIVE}

To compare the results of manual and automated White Blood Cell (WBC) counts in serous body fluids.

\section{METHODS}

A prospective study of 130 samples were performed manually using the improved Neubauer chamber with the same samples also being analysed on Sysmex XT-4000i.

\section{RESULTS}

It was observed that there is a statistically significant correlation between the values obtained in peritoneal and pleural fluids, but this is not the case with cerebrospinal fluid.

\section{CONCLUSIONS}

The Body Fluid Mode (BFM) of the Sysmex XT-4000i is a fast, reliable and accurate to count WBC's in body fluids.

\section{KEYWORDS}

Body-Fluid, Haematology Analyzer, Sysmex XT-4000i, Haemocytometer.

HOW TO CITE THIS ARTICLE: Parthasarathi UK, Prasad S, Jayaram, et al. Evaluation of automated cell counts in body fluids using Sysmex XT-4000i - an important need of the day! J. Evolution Med. Dent. Sci. 2016;5(72):5236-5239, DOI: $10.14260 /$ jemds/2016/1186

\section{INTRODUCTION}

The analysis of Body Fluids (BFs) is important in detecting signs of injury and infection as well as to aid in monitoring the status of diseased patients. ${ }^{1}$ Routine laboratory investigations of body fluids include physical, cytological, biochemical and bacteriological analysis which enable differentiation of the fluid between exudates and transudates and detection of infections and tumour cells. ${ }^{2}$ In Cerebrospinal Fluid (CSF), a WBC count of $>10$ cells $/ \mu \mathrm{L}$ in adults and $>10-30$ cells $/ \mu \mathrm{L}$ in children suggests meningitis or encephalitis, ${ }^{3}$ whereas in ascitic fluid spontaneous bacterial peritonitis is diagnosable when $>250$ Polymorphonuclear (PMNs) cells $/ \mu \mathrm{L}$ are counted. Cytological analysis comprises of microscopic examination, wherein the total and differential cell count is performed using a haemocytometer. ${ }^{2}$

In recent years, automated haematology analyzers with a Body Fluid Mode (BFM) are provided for the extension of its function in the analysis of cells in BFs. In this study, we evaluated the gold standard techniques of the improved

Financial or Other, Competing Interest: None.

Submission 02-08-2016, Peer Review 25-08-2016,

Acceptance 01-09-2016, Published 06-09-2016.

Corresponding Author:

Umadevi Komarla Parthasarathi,

Assistant Professor

Department of Pathology,

Aarupadai Veedu Medical College,

Puducherry.

E-mail: drumadevi16@gmail.com

DOI: $10.14260 /$ jemds/2016/1186
Neubauer chamber,and the counting of cells using an automated analyser, the Sysmex XT-4000i with BFM to count WBCs (Differential).

There is unfortunately very little literature available on this subject.

\section{MATERIALS AND METHODS}

The prospective study was done for 18 months using 130 samples of body fluids received at a diagnostic centre. The study samples included both paediatric and adult population. Fluids with coagulum, purulent and flocculent specimens were excluded from study and the samples were collected in EDTA or plain tubes. All samples were analysed within 2 hours of laboratory entry and stored between $4-6^{\circ} \mathrm{C}$ until the time of analysis after informed consent was obtained from the patients. For all the samples, the total WBCs and differential cell counts were performed, first manually by a haemocytometer, and secondly via automated analysis.

\section{Manual Method}

An improved Neubauer haemocytometer was used to count the total WBC's in the BF's. The WBC's were stained with WBC diluting fluid (Turks-water: glacial acetic acid: gentian violet =97:2:1) followed by counting of cells in the haemocytometer using 400x magnification after filling the counting chamber by means of a glass capillary (Fluid depth at $0.1 \mathrm{~mm}$ ). The total number of cells in the four large corner primary squares containing 16 smaller secondary squares each with area of $0.004 \mathrm{~mm}^{2}$ was counted. In cases where there was very low WBC count, all 9 primary squares were counted. Dilution of 
1:10 or $1: 20$ was used depending on the number of cells in specimen.

\section{Total WBC Count was Calculated as Follows}

$\mathrm{WBCs} / \mu \mathrm{L}=$ Total number of cells $\mathrm{x}$ dilution factor Volume

Manual differentiation was performed on cytospin samples after centrifugation, and the slides were stained by May-Grunwald-Giemsa. Differentiation of WBCs into PMNs (neutrophils, eosinophils, basophils) and mononuclear cells (MNs) (lymphocytes and monocytes) were determined.

\section{Automated Method}

Using automated haematology analyzer Sysmex XT-4000i with a specific BF mode, the total WBC and limited differential counts (PMNs and MNs) were determined. The equipment utilises flow cytometry and hydrodynamic focusing technologies to identify and cluster each cell type. Sample prepreparation is not required prior to its analysis. Analyzer aspirates $85 \mu \mathrm{L}$ of sample in open mode for one time analysis and uses nucleic acid dye, fluorescent light scatter (Size of cell) with side scatter (Inner complexity) light and fluorescence intensity (DNA/RNA concentration) to determine the total and limited differential counts in the 'DIFF' channel. Cells with increased fluorescence such as macrophages and mesothelial cells are also counted in DIFF scattergram. "WBC abnormal scattergram" flag will be generated when cells present in sample exceeds instrument cut-off value.

\section{Ethics}

The study was approved by the Institutional Review Board.

\section{Statistical Analysis}

All data analysis was performed using statistics software program (SPSS for the window - version 16.0). The cell counts obtained by the improved Neubauer chamber method and with the Sysmex XT-4000i were compared using the correlation coefficient. The correlation was considered significant when $\mathrm{P}<0.05$.

\section{RESULTS}

Of a total of 169 BF samples received, only 130 were used for the comparative study. Thirty-nine samples were excluded from the study due to technical difficulties such as the samples being inadequate, containing visible clot or subjected to improper storage methods. CSF constituted the majority 57 (44\%) of the samples followed by peritoneal 40 (31\%) and pleural fluids 33 (25\%) Figure 1.

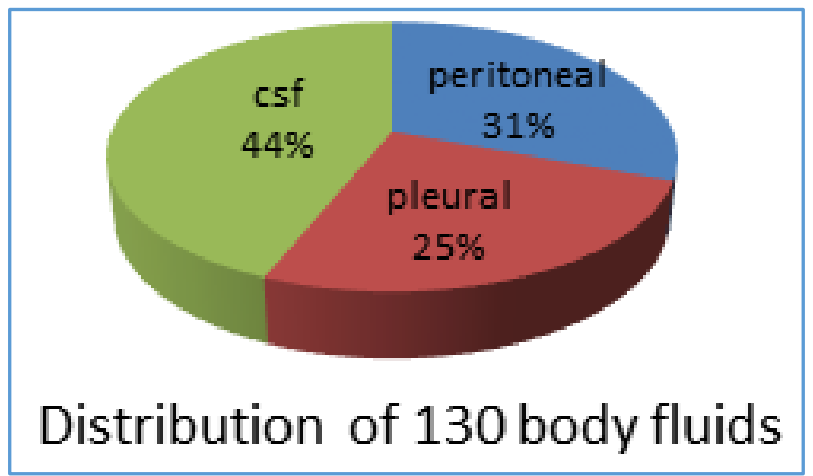

Fig. 1: Total Distribution of Body Fluid Samples
The patient's ages ranged from 23 days to 78 years, although the samples were predominantly from adults which accounted for 112 samples (86\%) and the rest $18(14 \%)$ samples were from the paediatric age group. The most common indication was to rule out infection, inflammatory condition and haemorrhage. The total WBC count from all the samples by the manual cell counting method range from 0 to 2984 cells $/ \mu \mathrm{L}$, whereas Sysmex counts were of the broader range of 0 to 3864 cells $/ \mu \mathrm{L}$. The correlation coefficient for the entire range of data (0.99) is statistically significant $(p=.000<0.001$, HS). The analyzer yielded higher cell counts than manual counts.

\section{Peritoneal Fluid}

The comparison between XT-4000i and microscopic analysis of 40 peritoneal fluids yielded considerable agreement. A significant positive correlation was found with the correlation coefficient being $0.98(\mathrm{p}=.000<.001, \mathrm{HS})$ Figure 2 .

The correlation with manual and automated method for differential cell count was also good with correlation coefficient of 0.79; whereas for macrophages and mesothelial cell count it was only 0.64 .

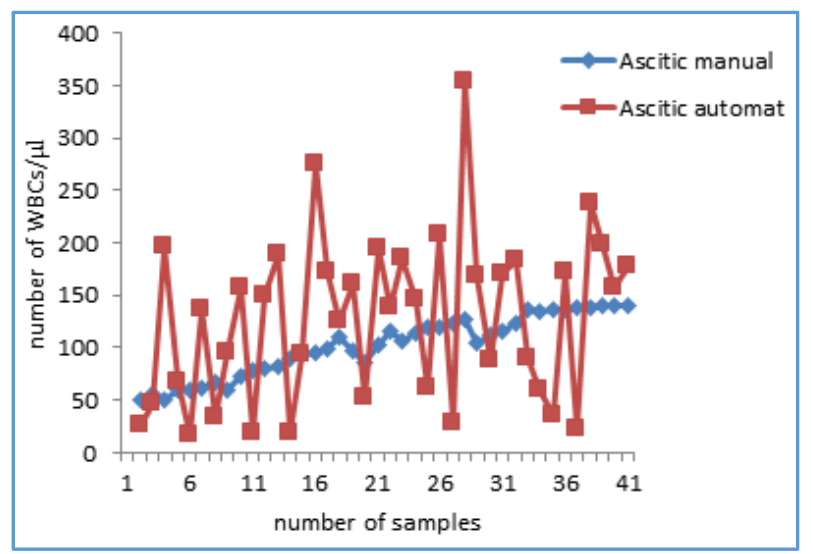

Fig. 2: Comparison between Manual and Automated White Blood Cells Count in Peritoneal Fluid

\section{Pleural Fluid}

Of 33 pleural fluid samples analysed, excellent agreement was found when counting WBCs by both the methods with a correlation coefficient being 0.96 ( $p=.000<.001$, HS) Figure 3.

When correlation of mesothelial cells and macrophages was assessed lower correlation was obtained (0.62) between the two methods. In addition, the differential cell counts obtained manually and with automated method also yielded lower correlation (0.58).

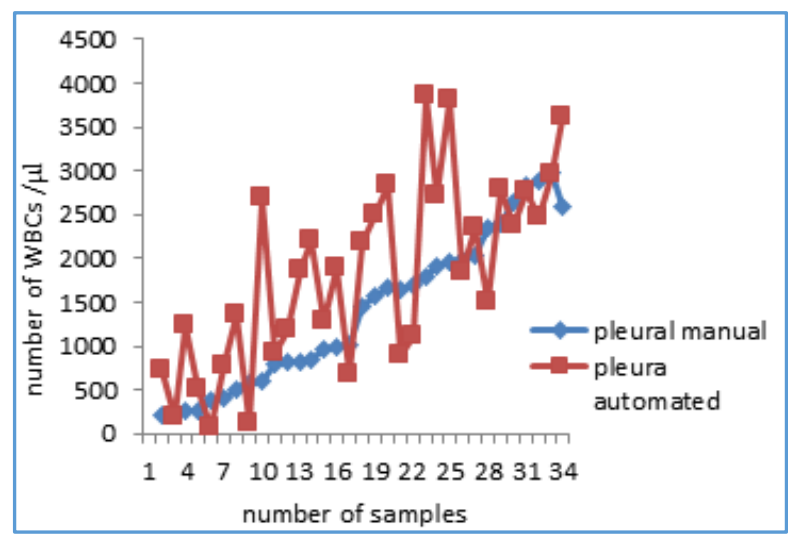

Fig. 3: Comparison between Manual and Automated White Blood Cells Count in Pleural Fluid 


\section{CSF}

It accounted for 57 samples (44\%), among which 40 samples were from the adult population and 17 from the paediatric age group. The most common indication for lumbar puncture was to rule out infection or other inflammatory condition. Very few samples were of diagnostic significance. The CSF samples were clear and colourless and with low cell counts. Manual cell counts ranged from $0-22$ cells $/ \mu \mathrm{L}$, while the automated method demonstrated $0-88$ cells $/ \mu \mathrm{L}$. Less agreement in results was observed when the cell counts by manual and automated methods were correlated. The correlation coefficient was 0.3 $(\mathrm{p}=0.08, \mathrm{NS})$ Figure 4 .

Samples with manual counts more than 15 cells/ $\mu \mathrm{L}$ were very less. Analyzer counts were higher than manual counts for large number of samples. Discordance between two methods tend to occur at very low manual counts $(<10$ cells $/ \mu \mathrm{L})$. Nineteen samples had very low manual cell counts, while 42 samples had counts with range from 11-22 cells/ $\mu \mathrm{L}$. Discordance in differential cell count by both methods was observed when sample contained benign CSF lining cells and organisms like Cryptococcus, which was noted in 8 and 1 case respectively.

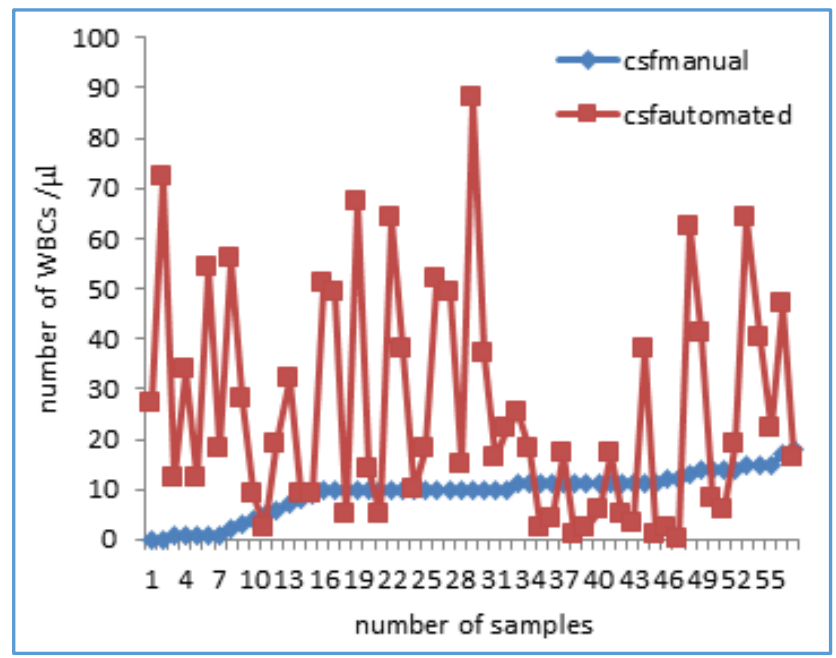

Fig. 4: Comparison between Manual and Automated White Blood Cells Count in Pleural Fluid

\section{DISCUSSION}

The analysis of BFs is an essential part of disease diagnosis, for follow-up action and differentiation of fluids into exudate and transudate. It also aids in the detection of infection and/or tumour cells. Therefore, the rapid and accurate results from BFs are of prime importance. Earlier, counting of white blood cells was performed by the use of a manual haemocytometer, but this method is disadvantaged by inter-observer variability, inaccuracy and that it is also time consuming. Presently, automated haematology analyzers are used with flow cytometry technology to count and differentiate cells easily and accurately, thereby reducing the turnaround time and increasing the precision of the results obtained. 4

Body fluids altogether have a different matrix than blood. Therefore, it is vital to ensure reliable results are produced by the analyzer for each type of BF. ${ }^{5}$ In our study, a total of 130 samples were included among which CSF samples were 57 (44\%) and constituted the majority followed by peritoneal, which accounted for 40 in number (31\%) and finally pleural fluid, which constituted 33 (25\%) cases. Total WBC counts with XT-4000i were accurate in all the BF samples when compared with chamber counting method. Samples analysed in XT-4000i yielded higher total cell counts than those with manual method. Similar results were observed by Denise et al, in which the comparison was done using three different automated analyzers. ${ }^{6}$ While in the study by Daikelly et al, the analyzer detected small numbers of cells when compared to manual counts. ${ }^{2}$ The correlation coefficient for the entire range of data was 0.99 . This closely agreed with the study conducted by Linda et al, in which she obtained a correlation coefficient of 0.77 with study sample constituting $187 \mathrm{BFs}^{7}$ In another study by Goubard et al, the correlation ranged from 0.82 to $0.96 .^{8}$ The study done by Paris A and Buoro et al, also showed similar results. ${ }^{3,9}$

Comparison of total WBCs manually and with XT-4000i in peritoneal fluids yielded excellent agreement with correlation coefficient being 0.98. Lippi and Paris et al acquired results akin to this in their study. ${ }^{3,8}$ When differential counts in peritoneal fluid was evaluated, clear samples showed a predominance of lymphocytes, and haemorrhagic samples showed a predominance of neutrophils. Complete agreement was found for both MNs and PMNs in peritoneal fluid, whereas lower correlation was observed between the two methods in differentiating cells in pleural fluid (0.58). Similar findings have been reported in the study by Barrett et al 10 On manual microscopy, the discordant cases showed the presence of reactive mesothelial cells and macrophages. Similar results were observed by Daikelly et al and attributed to the equipment's inherent limitations. ${ }^{2}$

CSF analysis of WBC counts by manual and automated methods showed a less agreement with correlation coefficient of 0.3. The same was observed by Paris and Linda et al.3,7 The number of cells counted in CSF samples were less and did not exceed 100 with both the methods when sample was clear. Whenever the cell count in BFs was observably low, there was a variation in the analyzer counts. We found less agreement in CSF samples between two methods at low end of the cell counts. Similar findings were observed by Linda et al attributed to the uncertainty of analyzer results when there are very low cell counts. ${ }^{7}$ Automation has low specificity in detecting cells in samples with very low counts $0-5$ cells $/ \mu \mathrm{L}$ accounting to the analyzer's lower limit for WBC linearity. Similar findings were observed by Linda and Zimmermann et al.7,11 False high counts are obtained, particularly with CSF samples which was attributed to carryover by previous test results. Similar findings was reported by Jonge et al. ${ }^{12}$

Tilmann et al noted that obtaining WBC counts using Sysmex equipment saves considerable time when compared with manual method of counting. ${ }^{13}$ Correlation of automated and manual PMN and MN cell counts in CSF samples were of limited value, because the study samples were of extremely low cell counts. It was difficult to draw conclusions about the analyzer's flagging capabilities, as the study population included in CSF was of low pathologic significance.

Analysis of BFs on haematology analyzers, such as Sysmex XT-4000i are capable of determining the total WBCs and differential with certain limitations.

\section{CONCLUSION}

The results of this study demonstrates that automated BF analysis using the Sysmex XT-4000i is an excellent alternative to manual counts in respect to peritoneal and pleural fluids 
and therefore can be used in routine laboratory analysis. However, this correlation is not so positive with CSF samples wherein they can generate false-positive or false-negative results, thereby stressing the importance of microscopic examination. For CSF result inference, further studies with large number of samples are required to draw a definite conclusion.

\section{REFERENCES}

1. Fleming C, Brouwer R, Lindemans J, et al. Validation of the body fluid module on the new Sysmex XN-1000 for counting blood cells in cerebrospinal fluid and other body fluids. Clin Chem Lab Med 2012;50(10):1791-8.

2. Braghirolli DI, Pranke P, Calil LN. Evaluation of semiautomated cells counting in peritoneal fluid. J Bras Patol Med Lab 2015;51(4):224-228.

3. Paris A, Nhan T, Cornet E, et al. Performance evaluation of the body fluid mode on the platform Sysmex XE-5000 series automated hematology analyser. Int J Lab Hematol 2010;32(5):539-47.

4. Aune MW, Becker JL, Brugnara C, et al. Automated flow cytometric analysis of blood cells in cerebrospinal fluid: analytic performance. Am J Clin Path 2004;121(5):690700.

5. Clinical and Laboratory Standards Institute. Body fluid analysis for cellular composition. Approved guideline: CLSI document H56-A, 950 West Valley Road, Suite 2500, Wayne, PA, 19087 USA, 2006.

6. Danise P, Maconi M, Rovetti A. Cell counting of body fluids: comparison between three automated haematology analysers and the manual microscope method. Int J Lab Hematol 2013;35(6):608-13.
7. Sandhaus LM, Ciarlini P, Kidric D, et al. Automated cerebrospinal fluid cell counts using the Sysmex XE-5000: is it time for new reference ranges? Am J Clin Pathol 2010;134(5):734-8.

8. Lippi G, Cattabiani C, Benegiamo A, et al. Evaluation of white blood cell count in peritoneal fluid with five different hemocytometers. Clin Biochem 2013;46 (1-2):173-6.

9. Buoro S, Gustinetti R, Dominoni P, et al. Analytical evaluation of Sysmex UF-1000i for flow cytometric analysis of peritoneal fluid. Clin Biochem 2012;45(15): 1263-5.

10. Conner BD, Lee YC, Branca P, et al. Variations in pleural fluid WBC count and differential counts with different sample containers and different methods. Chest 2003;123(4):1181-7.

11. Zimmermann M, Ruprecht $K$, Kainzinger $F$, et al. Automated vs. manual cerebrospinal fluid cell counts: a work and cost analysis comparing the Sysmex XE-5000 and the Fuchs-Rosenthal manual counting chamber. Int J Lab Hematol 2011;33(6):629-37.

12. De Jonge R, Brouwer R, De Graaf MT, et al. Evaluation of the new body fluid mode on the Sysmex XE-5000 for counting leukocytes and erythrocytes in cerebrospinal fluid and other body fluids. Clin Chem Lab Med 2010;48(5):665-75.

13. Kleine TO, Nebe CT, Lower C, et al. Cell analysis in cerebrospinal fluid (CSF) using Sysmex hematology analyzers XT-4000i and XE-5000: evaluation with CSF controls of the Joint German Society for clinical chemistry and laboratory medicine (DGKL). Cytometry A 2012;81(3):255-64. 